\title{
Trials and error
}

\author{
Tom Jacobs
}

Biotech drugmaker stock prices are volatile not just at quarterly earnings time, but throughout the heavily regulated drug development process. Early signs of a drug's success or failure can dramatically change investment potential versus risk, so intelligent investing requires deep knowledge of the process. Because the United States is the world's richest drug market - and thus often the first market targeted by a company with a promising biotech product-it's worth focusing exclusively on US clinical trials to illustrate some important take-home messages for biotech investors.

\section{The numbers}

A company that wants to sell a new (as opposed to generic) drug in the United States must receive marketing approval from the US Food \& Drug Administration (FDA). This happens-if it does-only after 10-15 years and hundreds of millions of dollars of development and testing. A big chunk (5-7 years, often even longer) of this time is taken up in human testing: 1.5 years for phase 1 (drug safety and proper dosage studies in $\sim 20-80$ healthy volunteers); an average of 2 years for phase 2 (efficacy and toxicity testing in 100-300 patients); and a whopping 3-4 years or more in phase 3 trials (efficacy confirmation and side-effect quantification in 1,000-3,000 patients). Such trials are often called pivotal trials for good reason: they generate the majority of data that determine whether the FDA will approve or disapprove a drug.

Investors should know the odds. Only 10\% of drugs that enter phase 1 secure approval, but as companies scuttle poor performers, that improves to $60 \%$ for drugs that enter phase 3 trials. This creates the dilemma: investing before phase 3 snares a better price but also more risk. Waiting provides more certainty but costs more.

Tom Jacobs is cofounder of Complete Growth Investor (http://www.completegrowth.com), a stock service for individual investors. He cannot give individual investment advice but welcomes your comments at tom@completegrowth.com.

\section{Improving the odds}

But no individual drug is average. A drug in phase 3 may have a $60 \%$ chance of success, but phase 3 can be longer or shorter-and don't forget the $40 \%$ risk of failure. To improve your chances, examine approval rates for drugs of a similar type. The FDA subjects new technology, such as using a monoclonal antibody to deliver a radioisotope, to greater scrutiny and is more likely to request more data than for a small molecule drug with a familiar mechanism of action. And if a drug is aimed at a condition for which another has been recently approved, the FDA sets the bar higher-the next candidate must be better, not just as good as its predecessor, so trial data must be stronger.

While trial data can be difficult for the nonexpert to fathom, the FDA provides some help. When the evidence is clear and positive, and/or a need for a drug so great, the FDA may agree to grease the wheels of the regulatory process. In such cases, they can grant a drug 'fast track' status, allowing a company 'rolling review' of the different parts of a drug's applicationeven before all the data has been submitted. If the drug is potentially a significant therapeutic advance over existing therapies, a drug may also obtain a 'priority review,' where the FDA aims to make a decision within six months of a new drug application's receipt. This is much better than the 12 months allotted under law. While positive, these guarantee only faster treatment, not approval, and problems can still surface at any time. Still, fast track status does improve the odds.

\section{Shifting goalposts}

After a company submits its application — either a New Drug Application (NDA) for a small molecule drug or a Biologics License Application (BLA) for a biotech drug-it presents the case to the FDA's outside expert advisory committees comprising $10-15$ physicians, nurses, statisticians, epidemiologists, and pharmacologists, expert in a particular area. They hold open hearings near the FDA's headquarters according to the schedule on the FDA's website (http:// www.fda.gov/oc/advisory). Because anyone can make an oral presentation after submitting some details, it can be a circus, but the meetings can show you how much controversy or consensus surrounds the drug, making it less or more likely that the full FDA will follow the Committee's recommendation.

After the Advisory Committee votes to recommend approval or disapproval, the FDA considers the committee recommendation and makes its decision. If it's positive the FDA may simply approve the drug for marketing as requested, or it may issue an approvable letter, saying that the drug is approvable subject to conditions such as resolving manufacturing issues, agreeing on the label and so on. The implication is that any issues are likely to be resolved.

Ultimately, the FDA may limit the label, which means to approve a drug for fewer indications (conditions or diseases) or types of patients than a company has applied for. Investors want the FDA to award the broadest label and thus broadest market possible. Although doctors do prescribe a drug off-label (that is, for an indication for which it was not approved) they are reluctant to so do. But even if the FDA does limit the label, a company may try to use postmarketing data or new trials to gain approval for a wider label encompassing a broader array of indications.

\section{Down, but not out}

Disapproval is not the last word either. A company and the FDA may discuss a better or different trial design, and a company may then commit significant money to continue trials. This can be a very positive sign for investors because, in effect, the company failed a test, talked over the reasons with the teacher and determined that it would likely do well on a retest with further study.

The most important point is that average results tell you nothing about a specific drug. If you know your company's drug trials well, you may avoid your own trials-and tribulations. 